\title{
Amélioration du procédé de fermentation traditionnelle des olives vertes
}

Latifa Bousmaha*, Mohammed El Yachioui et Mohammed Ouhssine UFR Amélioration et transformation microbienne et végétale, Laboratoire de biotechnologie microbienne, Département de biologie, Faculté des sciences de Kénitra, BPI33, Maroc.

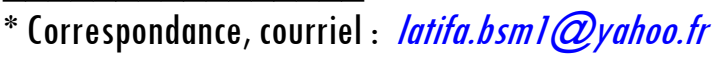

\section{Résumé}

Les levains de fermentation ont été utilisés depuis des siècles pour améliorer et favoriser la conservation des aliments. Le présent travail a pour but de mettre en évidence un procédé contrôlé fiable permettant la fermentation et la conservation des olives, pour remplacer le procédé traditionnel connu au Maroc, dont la qualité est souvent peu appréciable.

La bactérie lactique B2 et la souche de levure $\mathrm{Ll}$ isolées et sélectionnées, à grand pouvoir acidifiant et à haut potentiel fermentatif ont été retenues pour constituer le levain de fermentation. La fermentation des olives a été réalisée selon les trois protocoles expérimentaux suivants: une fermentation spontanée, une deuxième réalisée par un mélange simultané des deux souches (B2 et $\mathrm{Ll}$ ) et une troisième par une inoculation par la levure puis par la bactérie lactique après abaissement du pH. L'évolution de la fermentation a été suivie par analyse des paramètres physicochimiques (température, acidité, pH) et microbiologiques (FMAT, Coliformes, bactéries lactiques et levures). L'acidité a augmenté pour atteindre 1,1\%, le pH a baissé pour atteindre 3,9 en fin de la fermentation. Cette fermentation contrôlée a permis une réduction du temps de fermentation, l'obtention des olives de qualité homogène et de qualité hygiénique finale satisfaisante.

Mots-clés : olive, bactérie lactique, levure, fermentation contrôlée. 


\section{Abstract \\ Process improvement of traditional fermentation of green olives}

Olive fermentations were performed under the three following experimental process, olive naturally fermented and brine inoculated with a suitable microbial inoculum and concentration of salt.

This paper describes attempts to replace natural fermentations with fermentations conducted with a very small and suitable inoculum (only 2 categories of microorganisms).At the first time we used a yeast until the $\mathrm{pH}$ becomes slightly acid then the brine was inoculated by the lactic acid bacteria and at the second time we used a mixture of the 2 microorganisms. This is a report where the acidification of the brine was naturally done by the microbial inoculum.

The parameters measured were cells counts (yeasts, lactic acid bacteria, FMAT, coliforms), and contents of other organic acids, $\mathrm{pH}$ and temperature. The content of organic acids was increased with time and the $\mathrm{pH}$ dropped with time from 7,00 to 3,9. A good quality fruits with good savor and good texture were obtained. The microorganisms found in natural fermentations can be replaced by a judicious selection of strains.

Keywords : Olive, lactic bacterium, yeast, controlled fermentation.

\section{Introduction}

La conservation des légumes et fruits par fermentation remonte à l'antiquité et trouve son origine en orient. Ce procédé a longtemps été le principal mode de conservation dans différentes parties du monde. Appliquée essentiellement à la choucroute, aux concombres et aux olives, elle devrait également permettre de créer de nouveaux produits à partir d'autres légumes. Cette pratique est la richesse de différents pays à travers le monde en matière de nutrition[1-5].

Elle est utilisée pour la biopréservation des produits car elle augmente la durée du stockage ainsi que la qualité finale du produit en utilisant la microflore naturelle ainsi que les produits antibactériens produits [6]. L'olive fermentée peut être stockée pour une longue durée à raison de la teneur en acide lactique et l'industrialisation de la production d'olive au Maroc nécessiterait le remplacement du procédé traditionnel par un procédé initié par un nombre minimum de microorganismes. 
Aujourd'hui, avec $98 \%$ des oliviers qui existent dans le monde, le pourtour de la Méditerranée rassemble l'essentiel de la production mondiale d'huile d'olive. En outre de la Communauté Européenne qui est le premier consommateur mondial, les consommations les plus importantes dans le bassin méditerranéen se trouvent en Syrie, Turquie, Maroc et Tunisie. La consommation des olives au Maroc ne cesse de croître, en effet il s'agit d'un produit possédant un attrait indiscutable vu ses valeurs nutritives et ses caractéristiques organoleptiques très appréciées.

Les résultats obtenus par fermentation traditionnelle demeurent variables de point de vue qualité et la présence de proliférations microbiennes au niveau de ces produits est toujours notée. II serait donc intéressant de remplacer le procédé traditionnel dont les résultats sont imprévisibles par un procédé contrôlé initié par un nombre limité de microorganismes (une levure et une bactérie lactique) et avec lesquelles la fermentation serait plus rapide et de bonne qualité hygiénique.

Une vingtaine de levures et de bactéries lactiques impliqués dans les processus de fermentation traditionnelle des olives et des citrons ont été isolées, La levure sélectionnée était prédominante, résistante à $15 \%$ de sel et présente depuis le début de la fermentation spontanée du citron. La bactérie lactique B2 est choisie comme représentante du groupe homofermentaire et possédant un grand pouvoir acidifiant

\section{Matériel et méthodes}

\section{2-1. Procédure de saumurage des olives}

Les olives sont d'abord désamérisées par une solution de $\mathrm{NaOH}$ à $2 \%$ jusqu'à ce que la solution pénètre dans les $2 / 3$ du fruit, puis une série de lavages à l'eau courante est réalisée pour $72 \mathrm{~h}$. Ces olives sont mises ensuite en saumure à $15 \%$ de sel. Ces fermentations ont été réalisées a température ambiante variant de 16 à $24^{\circ} \mathrm{C}$.

\section{2-2. Choix des souches pour inoculation et réalisation de la fermentation contrôlée des olives}

Les microorganismes utilisés dans cette fermentation sont isolés à partir de la saumure de la fermentation traditionnelle des citrons. Seules les bactéries à gram positif et catalase négative ont été retenues et striées sur milieu De Man Rogosa et Sharp Agar (MRS). Les levures sont isolées sur milieu Potato dextrose agar (PDA) et les souches sont ensuite conservées à $4^{\circ} \mathrm{C}$. 
La levure choisie était prédominante, résistante à $15 \%$ de sel, et présente depuis le début jusqu'à la fin de fermentation.. La bactérie lactique retenue possède un fort pouvoir acidifiant.

Pour l'estimation du pouvoir acidifiant par mesure du $\mathrm{pH}$ final, la culture des bactéries lactiques est réalisée sur milieu MRS à pH initial de 5,88 et les levures sur milieu semi synthétique formé par le glucose $(3 \mathrm{~g} / \mathrm{L})$, l'extrait de levure $(3 \mathrm{~g} / \mathrm{L}), \mathrm{Mg} \mathrm{SO}_{4}$ $(\mathrm{lg} / \mathrm{L}), \mathrm{KH}_{2} \mathrm{PO}_{4}(\mathrm{lg} / \mathrm{L})$ et $(\mathrm{NH} 4)_{2} \mathrm{SO}_{4}(\mathrm{lg} / \mathrm{L})$ et à $\mathrm{pH}$ de 5,88 .

\section{2-3. Détermination des microorganismes du levain}

Les levures et les bactéries lactiques se succèdent selon leur ordre d'acidotolérance et d'adaptation au cours de la fermentation. Diverses successions ont été élaborés et une grande diversité écologique de levures et des bactéries lactiques dans les aliments est signalée dans différents écrits peu d'intérêt lors de ce travail a été élaboré à l'écologie microbienne. L'intérêt a été focalisé sur la sélection d'une souche de levure et de bactérie lactique à grand pouvoir acidifiant et à haut potentiel fermentatif pour remplacer le procédé traditionnel et garantir une bonne hygiène via l'acidité obtenue. La levure retenue appartient au genre Candida spp, la bactérie lactique au genre Lactobacillus spp.

\section{2-4. Protocole de la fermentation}

Des olives prises sur les marchés de Rabat ont été préparés comme décrit précédemment soigneusement après avoir éliminé les fruits endommagés et marinées dans une saumure à 15\%.. Les olives ont été manuellement ouvertes avec des couteaux stérilisés sur une surface stérilisée dans des conditions aseptiques. La fermentation des olives a été réalisée selon trois protocoles expérimentaux :

1-Des olives laissées à température ambiante fermenter spontanément.

2-Les olives sont inoculés dans un premier temps par la levure $\mathrm{Ll}$ à raison de $0,75 \%$ et une fois le pH atteint la valeur de 5,00 la bactérie lactique a été introduite dans la saumure à raison de $0,75 \%$.

3- Les olives sont inoculées simultanément par un mélange de la levure et de la bactérie lactique à raison de $1,5 \%$.

Une concentration de $3 g / L$ de glucose est additionnée à la saumure dans les deux cas. La concentration de $15 \%$ de sel est retenue après une étude préliminaire. 


\section{2-5. Echantillonage et suivi de la fermentation}

Des prélèvements à partir de la saumure des olives sont effectués périodiquement. Les paramètres mesurés sont: le pH, la température, l'acidité et la charge bactérienne (la FMAT, les coliformes et bactéries lactiques) ainsi que les levures.

L'énumération de la FMAT est réalisée sur milieu Plate Count Agar (PCA), coliformes sur milieu Desoxycholate Agar (DCL), les bactéries lactiques sur milieu De Man Rogosa et Sharp Agar (MRS) et les levures sur milieu Potato Dextrose Agar (PDA).

\section{Résultats et discussion}

Plusieurs souches microbiennes ont été isolées et qui ne sont pas toutes indispensables pour le processus de fermentation. En effet les souches se succèdent selon leur ordre d'acidotolérance et d'adaptation aux conditions de salinité régnant dans la saumure.

Tableau 1 : Screening des levures isolées de diverses fermentations végétales.

\begin{tabular}{|c|c|c|}
\hline $\begin{array}{l}\text { Levures isolés de la saumure } \\
\text { des citrons et olives en } \\
\text { fermentation spontanée }\end{array}$ & Saumure d'isolement & $\begin{array}{l}\text { pH obtenu après } 48 \mathrm{~h} \\
\text { d'incubation en milieu semi } \\
\text { synthétique, (pH initial } 5,88 \text { ) }\end{array}$ \\
\hline Ll & \multirow{12}{*}{ Citron } & 3,8 \\
\hline $\mathrm{L} 2$ & & 4,5 \\
\hline L3 & & 4,9 \\
\hline L4 & & 5,1 \\
\hline $\mathrm{L} 5$ & & 4,7 \\
\hline L6 & & 4,8 \\
\hline $\mathrm{L} 7$ & & 5,4 \\
\hline L8 & & 4,0 \\
\hline $\mathrm{L9}$ & & 4,9 \\
\hline $\mathrm{LI0}$ & & 5,3 \\
\hline LII & & 5,0 \\
\hline L12 & & 4,7 \\
\hline L13 & \multirow{5}{*}{ Olives } & 4,7 \\
\hline L14 & & 5,2 \\
\hline L15 & & 5,5 \\
\hline L16 & & 4,9 \\
\hline $\mathrm{LI7}$ & & 5,1 \\
\hline
\end{tabular}




\begin{tabular}{|c|c|c|}
\hline L18 & & 5,5 \\
\hline L19 & & 5,0 \\
\hline L20 & & 5,2 \\
\hline L21 & \multirow{12}{*}{ Carottes } & 4,0 \\
\hline L22 & & 3,9 \\
\hline L23 & & 4,8 \\
\hline L24 & & 5,5 \\
\hline L25 & & 4,5 \\
\hline L26 & & 4,2 \\
\hline L27 & & 5,1 \\
\hline L28 & & 4,6 \\
\hline L29 & & 4,5 \\
\hline L30 & & 5,0 \\
\hline L31 & & 4,8 \\
\hline L32 & & 4,6 \\
\hline
\end{tabular}

La levure choisie était la prédominante, résistante à $15 \%$ de sel et qui est présente pendant la fermentation naturelle du citron. la levure (LI) possédant un fort potentiel fermentatif est capable de baisser le pH de 5,88 à 3,6 après 48 heures d'incubation dans un milieu semi synthétique formé par le glucose $(3 \mathrm{~g} / \mathrm{L})$, l'extrait de levure $(3 \mathrm{~g} / \mathrm{L}), \mathrm{Mg} \mathrm{SO}_{4}(\mathrm{lg} / \mathrm{L}), \mathrm{KH}_{2} \mathrm{PO}_{4}(\mathrm{lg} / \mathrm{L})$ et $\left(\mathrm{NH}_{4}\right)_{2} \mathrm{SO}_{4}(\mathrm{lg} / \mathrm{L})$ à $30^{\circ} \mathrm{C}$ (Tableau $)$.

La bactérie lactique choisie B2 possède un fort pouvoir acidifiant (Tableav 2). Les bactéries lactiques jouent un rôle essentiel dans la conservation des aliments et sont largement utilisés en fermentations végétales. Ce sont les meilleurs microorganismes étudiés [7].

Des travaux antérieurs ont montré que la présence d'un représentant au moins des levures, des bactéries lactiques et des bactéries productrices d'acide acétique est nécessaire en fermentation [7].

Les bactéries lactiques tolèrent des hautes concentrations de sel; utilisent le glucose par la voie d'Embden-Meyerhoff-Parnas et produisent plus de 85\% d'acide lactique pour les souches homofermentaires. Cette tolérance au sel leur permet de commencer leur métabolisme et de produire de l'acide lactique recherché par les industriels et redouté par les microorganismes indésirables [8]. 
Tableau 2 : Screening des bactéries lactiques isolées de diverses fermentations végétales.

\begin{tabular}{|c|c|c|}
\hline $\begin{array}{l}\text { Bactéries lactiques isolées } \\
\text { de la saumure des citrons } \\
\text { et des olives en } \\
\text { fermentation spontanée }\end{array}$ & Saumure d'isolement & $\begin{array}{l}\text { pH obtenu après } 24 \mathrm{~h} \\
\text { d'incubation en milieu MRS } \\
\text { liquide à pH initial de } 5,88 \text {. }\end{array}$ \\
\hline $\mathrm{Bl}$ & & 3.18 \\
\hline B2 & & 3.02 \\
\hline B3 & & 3.38 \\
\hline B4 & & 3.65 \\
\hline B5 & & 3.87 \\
\hline B6 & & 4.03 \\
\hline B7 & & 3.48 \\
\hline B8 & & 4.03 \\
\hline B9 & & 3.48 \\
\hline B10 & & 3.98 \\
\hline BII & Citron & 3.93 \\
\hline $\mathrm{B} 12$ & & 3.85 \\
\hline $\mathrm{B} 13$ & & 4.52 \\
\hline B14 & & 5,01 \\
\hline B15 & Olives & 4,58 \\
\hline B16 & & 5,5 \\
\hline $\mathrm{B} 17$ & & 4,9 \\
\hline B18 & & 5,1 \\
\hline B19 & & 4,4 \\
\hline B20 & & 4,8 \\
\hline B21 & & 3,85 \\
\hline B22 & & 4,52 \\
\hline B23 & & 4,02 \\
\hline B24 & & 4,10 \\
\hline B25 & Carottes & 3,98 \\
\hline B26 & & 3,82 \\
\hline B27 & & 4,03 \\
\hline B28 & & 4,15 \\
\hline
\end{tabular}

\section{3-1. En culture successive}

\section{3-1-1. pH et acidité}

En culture successive, la saumure à pH neutre 7,00, une fois inoculée par la levure devient légèrement acide $(5,5)$ après 4 jours de fermentation. Une fois le pH devient 
acide 5,00, la bactérie lactique B2 est inoculée ce qui permet la chute du pH de 5,0 à 4,3 après 28 jours de fermentation (Figure ). L'acidité augmente pour atteindre $0,7 \%$ en fin de fermentation. Le produit obtenu est stable après la fin de la fermentation. En général les $\mathrm{pH}$ obtenus en fin de fermentations varient selon le végétal pour le Tarhana 3,4 [8] pour le khamir 3,9 [9] et Pour les olives 4,2 [10]. Le pH varie aussi selon le procédé appliqué aux olives c'est ainsi qu'un pH de 4,2 est obtenu au bout de 28 jours après inoculation de la saumure par lactobacillus plantarum et Pediococcus spp [10]. Des travaux récents [11] ont montré que l'acidification de la saumure avec $2 \%$ d'acide lactique et l'addition de $25 \%$ d'HCL IN à la saumure ainsi que la substitution de $20 \%$ de la saumure par une ancienne saumure a permis d'obtenir un pH de 4,8. Pour d'autres Lactobacillus plantarum reste toujours l'espèce dominante et qui a été utilisé comme starter à une concentration de $10^{7} \mathrm{UFC} / \mathrm{mL}$ suspendu en milieu MRS et à $4 \%$ de sel et une correction du pH initial avec l'acide acétique ce qui provoque une chute de $\mathrm{pH}$ et une acidification rapide durant les 25 jours après saumurage [12]. L'inoculation de la saumure par Lactobacillus plantarum, et la correction du $\mathrm{pH}$ ont permis de réduire le temps de fermentation ainsi que la flore de détérioration dans le cas des olives vertes Marocaines [13]. Pour d'autres la concentration de $6 \%$ de sel serait la concentration idéale pour une bonne acidification et prolifération des bactéries lactiques [14].

L'acidité varie aussi selon les végétaux de2,3\% pour la choucroute [15] de 0,6 à $0,8 \%$ pour les olives [10-16] de 1,7 à $2,3 \%$ et $1,6 \%$ pour le khamir [11] $1.1 \%$ pour le citron[17].

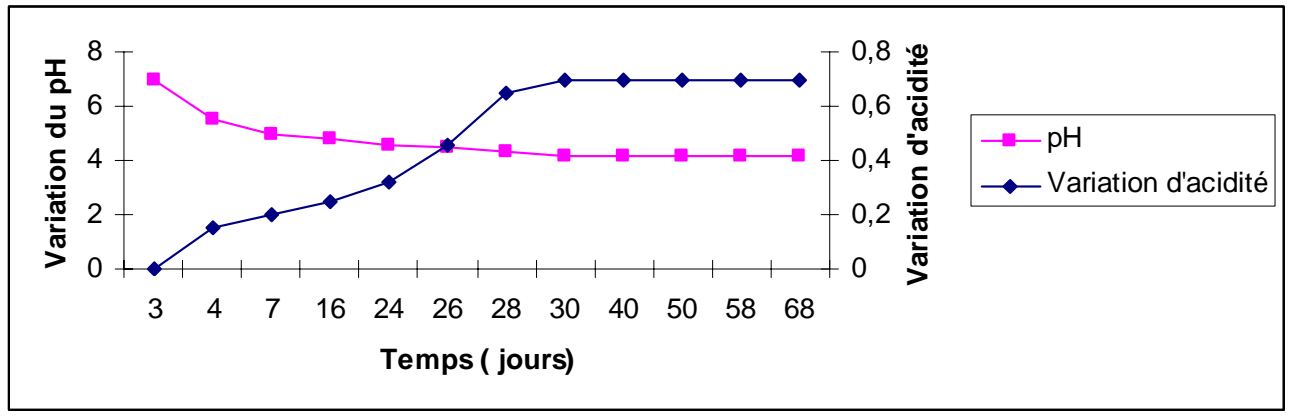

Figure 1 : Variation du pH et d'acidité au cours de la fermentation contrôlée des olives en culture successive.

\section{3-1-2. Evolution microbiologique}

L'évolution de la FMAT est irrégulière et l'absence des coliformes est notée après 7 jours de fermentation. Leurs présence à un taux $2,5.10^{3} \mathrm{Ufc} / \mathrm{mLest}$ du au $\mathrm{pH}$ 
légèrement élevé de 5,5 au début de fermentation.(Tableau 3) En effet certains travaux ont montré que les coliformes sont présents dans les aliments une fois la concentration en sel est inférieur à $5 \%$ ou si le pH est supérieur à 4,8 (Vaughn 1985) et c'est ainsi que les saumures doivent être renforcées par ajout de sel ou par acidification. L'acidification est réalisée de manière naturelle par l'acide lactique produit par la bactérie lactique choisie et à métabolisme homofermentaire et par les acides produits éventuellement par la levure retenue.

La levure est présente depuis l'inoculation jusqu'a la fin du suivi ce qui prouve que la levure est adaptée aux conditions de salinité de 15\% La bactérie lactique B2 est présente à taux variable tout le long du procédé de fermentation. Le pH stable obtenu après 28 jours est du à la dégradation complète des sucres présents dans la saumure de fermentation.

Tableau 3 : Evolution de la charge microbienne au cours de la fermentation dirigée des olives en culture successive

\begin{tabular}{|c|c|c|c|c|}
\hline Temps (jours) & FMAT & $\begin{array}{c}\text { Coliformes } \\
\text { (UFC/mL) }\end{array}$ & $\begin{array}{c}\text { Bactéries lactiques } \\
\text { (UFC/mL) }\end{array}$ & Levures \\
4 & $2,16.10^{4}$ & $2,5.10^{2}$ & 0 & $2,3.10^{4}$ \\
7 & $2,36.10^{4}$ & $1,5.10^{2}$ & 0 & $2,16.10^{5}$ \\
16 & $1,86.10^{3}$ & 0 & $4.10^{3}$ & $1,86.10^{5}$ \\
24 & $1,7.10^{3}$ & 0 & $4,5.10^{4}$ & $1,9.10^{5}$ \\
26 & $1,6.10^{4}$ & 0 & $4,7.10^{4}$ & $1,96.10^{4}$ \\
28 & $1,4.10^{4}$ & 0 & $5,3.10^{3}$ & $1,9.10^{4}$ \\
30 & $1,37.10^{3}$ & 0 & $5.10^{3}$ & $1,98.10^{4}$ \\
50 & $1,2.10^{4}$ & 0 & $4,7.10^{3}$ & $1,92.10^{4}$ \\
58 & $1,18.10^{4}$ & 0 & $3,8.10^{3}$ & $1,89.10^{4}$ \\
68 & $1,06.10^{4}$ & 0 & $3,7.10^{3}$ & $1,8.10^{4}$ \\
& & & & \\
\hline
\end{tabular}

\section{3-2. En culture mixte}

\section{3-2-1. pH et acidité}

L'utilisation du ferment mixte a permis une chute plus ou moins rapide du $\mathrm{pH}$, un $\mathrm{pH}$ de 4, 8 est obtenu après 7 jours de fermentation, 3,9 après 28 jours de fermentation. Une fois les sucres épuisées le pH devint stable. L'acidité augmente progressivement pour atteindre 1,1\% après 28 jours de fermentation (figure 2). 


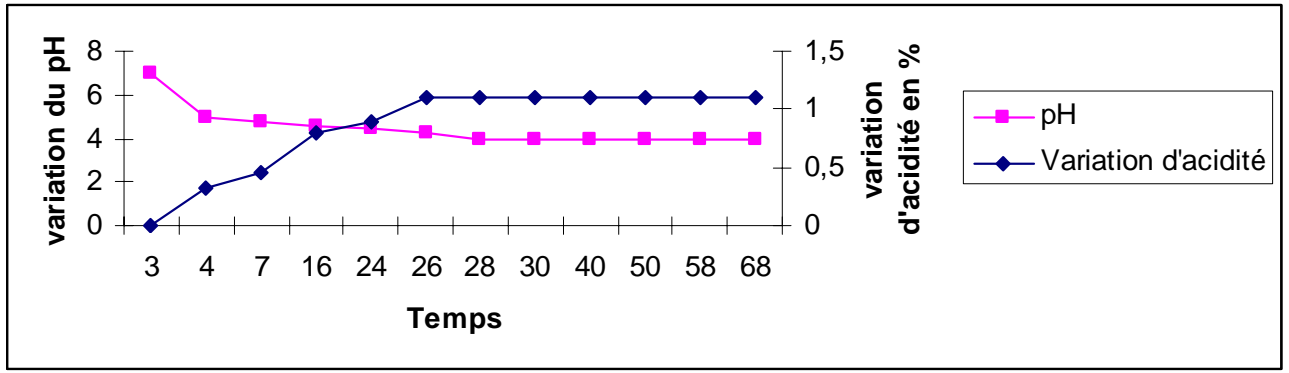

Figure 2: Variation du pH et d'acidité au cours de la fermentation contrôlée des olives en culture mixte.

\section{3-2-2. Evolution microbiologique}

L'évolution de la FMAT obtenue est irrégulière, l'absence des coliformes est aussi signalée après 7 jours de fermentation, la présence à un taux de $1,5.10^{2} \mathrm{UFC} / \mathrm{mL}$ moindre que celui enregistré dans le cas de la culture successive laisse suggérer que cette diminution serait du à la présence de la bactérie lactique ensemencée en mixture avec la levure. La présence de la levure et de la bactérie lactique est notée à taux variable (tableau 4).

Tableau 4 : Evolution microbiologique au cours de la fermentation contrôlée des olives en culture mixte

\begin{tabular}{|l|c|c|c|c|}
\hline $\begin{array}{l}\text { Temps } \\
\text { (Jours) }\end{array}$ & $\begin{array}{c}\text { FMAT } \\
\text { (UFC/mL) }\end{array}$ & $\begin{array}{c}\text { Coliformes } \\
\text { (UFC/mL) }\end{array}$ & $\begin{array}{c}\text { Bactéries lactiques } \\
\text { (UFC/mL) }\end{array}$ & Levures \\
4 & $1,89.10^{4}$ & $1,5.10^{2}$ & $1,9.10^{4}$ & $2,8.10^{4}$ \\
7 & $1,7.10^{4}$ & $1.10^{2}$ & $2,2.10^{5}$ & $3,2.10^{5}$ \\
16 & $1,8.10^{4}$ & 0 & $1,76.10^{5}$ & $2,35.10^{5}$ \\
24 & $1,7.10^{3}$ & 0 & $7,6.10^{3}$ & $2,75.10^{5}$ \\
26 & $1,87.10^{3}$ & 0 & $5.10^{3}$ & $1,8.10^{5}$ \\
28 & $1,7.10^{3}$ & 0 & $4,7.10^{3}$ & $1,76.10^{4}$ \\
30 & $1,65.10^{3}$ & 0 & $4,0.10^{3}$ & $1,6.10^{4}$ \\
50 & $1,6.10^{3}$ & 0 & $4,2.10^{3}$ & $1,9.10^{4}$ \\
58 & $1,63.10^{3}$ & 0 & $3,7.10^{3}$ & $1,65.10^{4}$ \\
68 & $1,6.10^{3}$ & 0 & $3,4.10^{3}$ & $1,58.10^{4}$ \\
& & & & \\
\hline
\end{tabular}




\section{Conclusion}

La fermentation spontanée ou la croissance non contrôlée de microorganismes dans des fruits s'accompagne de formation de gaz et de changements organoleptiques qui détériorent la qualité des produits. L'utilisation de l'inoculum a permis d'atteindre des valeurs appréciables de $\mathrm{pH}$ et d'acidité : La culture mixte préférable a la culture successive a permis d'atteindre un taux d'acidité de $1,1 \%$ et un pH de 3,9 après 28 jours de fermentation ce qui suggère le choix judicieux des deux souches.

\section{Références}

[1] - Steinkraus KH.. Lactic acid fermentation in the production of foods from vegetables, cereals and legumes. Antonie Van Leeuwenhoek. 49, (1983) 337-348.

[2] - Gadaga T. H., Mutukumira. A. N., Narvhus. J.A, et S. B. Feresu S. B.. A review of traditional fermented foods and beverages of Zimbabwe International Journal of Food Microbiology, 53, 1, (1999)1-11.

[3] - Desmazeaud M.. Les bactéries lactiques dans l'alimentation humaine ; utilisation et innocuité, cahiers "agricultures".5, (1996) 331-342

[4] - Caplice E., FitZGerald G.f.. Food fermentations, role of microorganisms in food production and preservation. J. Food.Microbial. 50, (1999) $131-49$.

[5] - Martin R. Adams.. Safety of industrial lactic acid bacteria, journal of biotechnology. 68, 2-3, (1999) 171- 178.

[6] - Stiles Me.. Biopreservation by lactic acid bacteria. Antonie Van leeunwenhoek. 70, (1996) 331-345.

[7] - will N Konings, Jan Kok, Oscar P Kuipers, Bert Poolman .. Current opinion microbiology, review article. 3, (2000) 276

[8] - Schwan R. F. Cocoa fermentations conducted with a defined microbial cocktail inoculum. Applied Environmental Microbiology. 64, (1998) 1477-1483.

[9] - Yves Romeo, Jean Bouvier and Claude Gutierrez. La réponse au stress osmotique des bactéries lactiques Lactococcus lactis et Lactobacillus plantarum. Lait. 81, (2001) 49-55

[10] - Daglioglu 0.. Tarhana as a traditional Turkish fermented cereal food, its recipe, production and composition, Nahrung. 44, (2000) 85-88.

[11]- Gassem MA.. Study of the micro-organisms associated with the fermented bread (khamir) produced from sorghum in Gisan region, Saudi Arabia; J Appl Microbial. 86, (1999) 221-225. 
[12] - - Mohieddine S.. Etude des altérations des olives fermentées, thèse de doctorat, Université Benmsik. Casablanca (1997).

[13]- Efstathios Z. Panagou et Constantinos $Z$. Katsaboxakis.. Effect of different brining treatments on the fermentation of cv. Conservolea green olives processed by the Spanish-method. Food Microbiology. 23, 2, (2005) 199-204.

[14] - ega M, J.L Ruiz -Barba, A.H Sanchez, L.Rejano, R. Jimenez- Diazand A Garrido.. Fermentation profile and optimization of green olive fermentation using lactobacillus plantarum LPC 010 as starter culture. Food Microbiology.20, 4, (2003) 421-430.

[15] - Lamzira Zahra, Abdeslam Asehraou, Dulce Brito, Manuella Oliveira2, Mohamed Faid et Cidalia Peres,. Reducing the Bloater Spoilage During Lactic Fermentation of Moroccan Green Olives .Food Technol. Biotechnol. 43 , 4, (2005) 373-375

[16] - Tassou C.C., Panagou E. Z., Katsaboxakis K. Z., 2004. Microbiological and physicochemical changes of naturally black olives fermented at different temperatures and $\mathrm{NaCl}$ levels in the brines. Food Microbiol. 19 (2002) 605-615.

[17] - Tamouh,. fermentation du citron connaissance et maîtrise. Thèse de doctorat, faculté chaiib Doukali. El jadida. (1996)

[18] - Vaughn R.H.,. The microbiology of vegetable fermentations: vol I, edited by B.J.B ;(1985) $49-110$. 